\title{
Proteome-wide prediction of targets for aspirin: New insight into the molecular mechanism of aspirin
}

Shao-Xing Dai, Wen-Xing Li, Gong-Hua Li, Jing-Fei Huang

Besides its anti-inflammatory, analgesic and anti-pyretic properties, aspirin is used for the prevention of cardiovascular disease and various types of cancer. The multiple activities of aspirin likely involve several molecular targets and pathways rather than a single target. Therefore, systematic identification of these targets of aspirin can help us understand the underlying mechanisms of the activities. In this study, we identified 23 putative targets of aspirin in the human proteome by using binding pocket similarity detecting tool combination with molecular docking, free energy calculation and pathway analysis. These targets have diverse folds and are derived from different protein family. However, they have similar aspirin-binding pockets. The binding free energy with aspirin for newly identified targets is comparable to that for the primary targets. Pathway analysis revealed that the targets were enriched in several pathways such as vascular endothelial growth factor (VEGF) signaling, Fc epsilon RI signaling and arachidonic acid metabolism, which are strongly involved in inflammation, cardiovascular disease and cancer. Therefore, the predicted target profile of aspirin suggests a new explanation for the disease prevention ability of aspirin. Our findings provide a new insight of the magical aspirin and its efficacy of disease prevention in a systematic and global view. 
1 Proteome-wide Prediction of Targets for Aspirin: New Insight into the Molecular Mechanism of Aspirin

3 Shao-Xing Dai ${ }^{1,2}$, Wen-Xing Li ${ }^{1,3}$, Gong-Hua $\mathrm{Li}^{1,2, *}$, and Jing-Fei Huang ${ }^{1,2,4,5, *}$

41 State Key Laboratory of Genetic Resources and Evolution, Kunming Institute of Zoology,

5 Chinese Academy of Sciences, Kunming 650223, Yunnan, China

62 Kunming College of Life Science, University of Chinese Academy of Sciences, Beijing

7 100049, China

83 Institute of Health Sciences, Anhui University, Hefei 230601, Anhui, China

94 KIZ-SU Joint Laboratory of Animal Models and Drug Development, College of 10 Pharmaceutical Sciences, Soochow University, Kunming 650223, Yunnan, China

115 Collaborative Innovation Center for Natural Products and Biological Drugs of Yunnan,

12 Kunming 650223, Yunnan, China

$13{ }^{*}$ Corresponding author:

14 Gong-Hua Li (ligonghua@mail.kiz.ac.cn)

15 Jing-Fei Huang (huangjf@mail.kiz.ac.cn)

16 Tel: +86087165199200

17 Fax: +86087165199200 


\section{Abstract}

Besides its anti-inflammatory, analgesic and anti-pyretic properties, aspirin is used for the prevention of cardiovascular disease and various types of cancer. The multiple activities of aspirin likely involve several molecular targets and pathways rather than a single target. Therefore, systematic identification of these targets of aspirin can help us understand the underlying mechanisms of the activities. In this study, we identified 23 putative targets of aspirin in the human proteome by using binding pocket similarity detecting tool combination with molecular docking, free energy calculation and pathway analysis. These targets have diverse folds and are derived from different protein family. However, they have similar aspirin-binding pockets. The binding free energy with aspirin for newly identified targets is comparable to that for the primary targets. Pathway analysis revealed that the targets were enriched in several pathways such as vascular endothelial growth factor (VEGF) signaling, Fc epsilon RI signaling and arachidonic acid metabolism, which are strongly involved in inflammation, cardiovascular disease and cancer. Therefore, the predicted target profile of aspirin suggests a new explanation for the disease prevention ability of aspirin. Our findings provide a new insight of the magical aspirin and its efficacy of disease prevention in a systematic and global view. 


\section{Introduction}

Aspirin, also known as acetylsalicylic acid, is a nonsteroidal anti-inflammatory drug (NSAID). The primary molecular mechanism of aspirin is the selective acetylaton of Ser-530 of cyclooxygenase-1 (COX-1) (Alfonso et al. 2014; Dovizio et al. 2013; Ghooi et al. 1995; Vane 1971; Vane \& Botting 2003), thereby inhibiting prostaglandin synthesis. This was the basis for its anti-inflammatory, antipyretic, and analgesic effects (Vane \& Botting 2003). In addition, recent studies revealed that phospholipases A2 (PLA2) is functionally coupled with cyclooxygenase- 1 and 2 pathway and part of its anti-inflammatory effects of aspirin may be due to its binding with PLA2 (Balsinde et al. 1999; Singh et al. 2005; Touqui \& Alaoui-El-Azher 2001).

0

Besides its the anti-inflammatory, analgesic, and anti-pyretic properties, aspirin is used for the prevention of cardiovascular disease and various types of cancer (Alfonso et al. 2014; Dovizio et al. 2013). Aspirin prevents myocardial infarction, and ischemic stroke when used in the primary prevention of cardiovascular disease (Berger et al. 2008; Nemerovski et al. 2012; Raju et al. 2011; Schnell et al. 2012; Younis et al. 2010). Furthermore, aspirin is also highly effective in preventing several common cancers (Avivi et al. 2012; Burn et al. 2011; Hassan et al. 2012; Rothwell et al. 2010; Rothwell et al. 2012; Thun et al. 2012). Taking aspirin daily reduced risk of distant metastasis by $30-40 \%$ and reduced the risk of metastatic adenocarcinoma by almost a half (Rothwell et al. 2012). Although it has been convincingly shown that aspirin can prevent cardiovascular disease and several cancer types, the molecular mechanisms underlying these effects of aspirin are not entirely clear. The multiple activities of aspirin cannot be attributed wholly to a single target and most likely involve several molecular targets and pathways (Deng \& Fang 2012; Din et al. 2012; Sclabas et al. 2005; Singh et al. 2005). Therefore, systematic identification of molecular targets of aspirin can help in understanding the 
84

85

86

87

mechanisms underlying the activities and adverse reactions of aspirin. Unfortunately, studies on the proteome-wide target profile of aspirin are very limited.

In this study, we predicted the targets of aspirin whole proteome-wide by combining structural bioinformatics and systems biology approaches. Starting with the binding sites of aspirin (BSiteAs), the potential targets of aspirin were discovered by using contact matrix based local structural alignment algorithm (CMASA) which was developed in our lab (Li \& Huang 2010). Then molecular docking and free energy calculation were applied to filter the improper targets to which the aspirin can not bind. We also analyzed the diversity of the putative targets and binding modes of aspirin. Finally, we performed the pathway analysis for the putative targets. We found several new targets for aspirin which are enriched in the pathways that are strongly involved in inflammatory, cardiovascular disease and cancer, such as vascular endothelial growth factor (VEGF), mitogen-activated protein kinase(MAPK), Fc epsilon RI signaling and arachidonic acid metabolism signaling pathways.

\section{Methods}

\section{Overview of pipeline for proteome-wide prediction of aspirin targets}

The pipeline for proteome-wide prediction of aspirin targets is outlined in Figure 1. Firstly, we constructed the structural database of human proteins (17425 non-redundant structures), and the binding sites of aspirin (BSiteAs) were used to search against this database using the program CMASA. Secondly, the binding abilities of aspirin to these putative off-targets were estimated using molecular docking. If aspirin docked unsuccessfully to the predicted binding pocket of a particular protein, this protein was removed from the target list of aspirin. Thirdly, the remaining putative targets were subject to molecular mechanics Poisson-Boltzmann surface area (MMPBSA) free energy calculation. Finally, we performed the pathway enrichment analysis for these putative targets.

\section{The structure database of human proteins}

The query database was built by integrating three available sources of protein structure. The first source is the Protein Data Bank managed by Research Collaboratory for Structural Bioinformatics (RCSB PDB) (http://www.rcsb.org) (Rose et al. 2011), which contains 6983 structures of human proteins. The other two repositories are structure models built by homology modeling techniques from Structure Atlas of Human Genome(Motono et al. 2011) 
114 (http://bird.cbrc.jp/sahg) and GPCR Research Database (GPCR-RD) (Zhang \& Zhang 2010),

115 which have 16529 and 1028 structures, respectively. This procedure yielded a total of 24540

116 human protein structures. The three available sources of protein structure were integrated and

117 removed the redundancy using CD-HIT Suite (Huang et al. 2010). The identity cut-off was set to

118 0.95. There are totally 17425 structures in our self-built 'non-redundant' structural database of 119 human proteins. The structural database represents a relatively complete and accurate library of 120 human proteins.

121 To detect and compare the aspirin binding sites in the structure database

122 We used all representative aspirin-protein complexes in our study. There are a total of six 123 aspirin-protein complex structures currently available (1OXR, 1TGM, 2QQT, 3GCL, 4NSB and 124 3IAZ). Both 1OXR and 1TGM are structures of phospholipase A2, we only used 1OXR in this 125 study (Figure 2A). 2QQT and 3GCL are bovine lactoperoxidase, and we used 3GCL in this 126 study. 4NSB and 3IAZ are structures of buffalo chitinase-3-like protein 1 and bovine 127 lactotransferrin, respectively. The two structures were also used in our study. In addition, 1PTH, 128 the complex structure of salicylic acid and COX-1, was also used in this study (Figure 2A). 129 Therefore, a total of five complex structures (1OXR, 3GCL, 4NSB, 3IAZ and 1PTH) were used in this study. The binding sites of aspirin (BSiteAs) were defined as amino acid residues lying within $5 \AA$ from atoms of the aspirin. The BSiteAs were used to search against 17425 nonredundant structures using the program CMASA (http://bsb.kiz.ac.cn/CMASA_flex) which was developed in our lab. The accuracy and sensitivity of this tool were 0.96 and 0.86 , respectively. The workflow of CMASA was as following. First, the CMASA parsed the query binding site and the database to be searched. Second, for each structure in database, based on the query binding site, the CMASA enumerated all possible combination of residues in the structure using amino acid substitute. The blocks substitution matrix 62 (BLOSUM62, cutoff=1) was used. These residue combinations are similar to that in the query binding site. Each residue combination forms a possible local structure. Third, the CMASA used contact matrix average deviation (CMAD) between the query binding site and these local structures to filter the candidate matches. Then, the CMASA calculated the RMSD and the RMSD based P-value if the CMAD < cutoff. At last, the CMASA ranked all of the hits and add their information. Hits are considered significant if the CMASA E-value $<0.01$.

\section{Molecular docking}

The hit proteins have similar local structures with BSiteAs and potential to bind to aspirin. 
146 However, it does not mean that aspirin can certainly bind to these proteins. To assess whether

147 aspirin can bind to these proteins, flexible ligand-rigid protein docking was performed using 148 CHARMm-based DOCKER (CDOCKER) (Wu et al. 2003) in the Discovery Studio v3.1. The 149 following steps are included in the CDOCKER protocol. 1) A set of ligand conformations are 150 generated using high-temperature molecular dynamics with different random seeds. 2) Random orientations of the conformations are produced by translating the center of the ligand to a specified location within the receptor active site, and performing a series of random rotations. A softened energy is calculated and the orientation is kept if the energy is less than a specified threshold. This process continues until either the desired number of low-energy orientations is found, or the maximum number of bad orientations have been tried. 3) Each orientation is subjected to simulated annealing molecular dynamics. The temperature is heated up to a high temperature then cooled to the target temperature. 4) A final minimization of the ligand in the rigid receptor using non-softened potential is performed. 5) For each final pose, the CHARMm energy (interaction energy plus ligand strain) and the interaction energy alone are calculated. The poses are sorted by CHARMm energy and the top scoring (most negative, thus favorable to binding) poses are retained. In this study, we generated 10 random conformations for each ligand. The parameters of the dynamic steps, the dynamic target temperature and include electrostatics are set to 1000 steps, $1000 \mathrm{~K}$ and True, respectively, which is the default setting in CDOCKER. The binding sphere of CDOCKER is defined around the local structure detected by CMASA. The center of binding sphere is set as the center of the local structure. And the radius of binding sphere is set as $10 \AA$, which allows the free rotation of aspirin. If aspirin docked successfully to a particular protein, the binding poses of aspirin showing the lowest energy were retained and used for MM-PBSA (Kollman et al. 2000; Kuhn et al. 2005) free energy calculation. The 3-D structures of docked complexes were visualized using PyMol v1.5.

\section{MM-PBSA free energy calculation and entropy change estimation}

171 The complex structures of aspirin and putative targets were further used to obtain more accurate estimate of binding free energy. Binding free energies $\left(\Delta \mathrm{G}_{\mathrm{bind}}\right)$ of aspirin at the binding site on these target proteins were calculated by using the MM-PBSA free energy calculation protocol in

174 Pipeline pilot v8.5 (http://accelrys.com/products/collaborative-science/biovia-pipeline-pilot/) as 175 follows :

176

$\Delta \mathrm{G}^{\mathrm{PB}}$ binding $=\mathrm{G}_{\text {complex }}-\mathrm{G}_{\text {receptor }}-\mathrm{G}_{\text {ligand }}$ 
177 where $\mathrm{G}_{\text {complex }}, \mathrm{G}_{\text {receptor}}, \mathrm{G}_{\text {ligand }}$ are the free energies of the complex, receptor and ligand 178 respectively. The free energy of each molecule on the right hand side can be considered as the

179

180

181

182

183

184

185

186

187

188

189

190

191

192

193

194

195

196

197

198

199

200

201

202

203

204

205

206

sum of molecular mechanics energy in gas phase $\left(\mathrm{E}_{\mathrm{MM}}\right)$ and solvation free energy $(\Delta \mathrm{sol})$.

As the entropy change (T $\Delta \mathrm{S})$ is the most time-intensive part, it was not included in the above calculation. We estimate the entropy change in another way. In the study of Chang \& Gilson (Chang \& Gilson 2004), they computed the entropy changes for different receptors upon same ligand binding. The results showed the entropy changes for different receptors were similar. Therefore, we can estimate an approximate entropy change. The process of entropy change estimation is as following: 1) Based on the formula: $-\mathrm{RT} \ln \mathrm{K}=\Delta \mathrm{G}=\Delta \mathrm{H}-\mathrm{T} \Delta \mathrm{S}$ (where $\mathrm{R}$ is the gas constant, $\mathrm{T}$ is the absolute temperature), the entropy changes (T $\Delta \mathrm{S})$ is equal to the sum of $\Delta \mathrm{H}$ and RTln K. 2) The experiment study have shown that aspirin binds to PLA2 enzyme (PDBID: 1OXR) specifically with a binding constant (K) of $1.56 \times 10^{5} \mathrm{M}^{-1}$ (Singh et al. 2005). For this complex, the enthalpy change $(\Delta \mathbf{H})$ was calculated as $-2.327 \mathrm{kcal} / \mathrm{mol}$ using the MMPBSA and RTln K was calculated as $7.055 \mathrm{kcal} / \mathrm{mol}$. So the entropy change $(\mathrm{T} \Delta \mathrm{S})$ is 4.728 $\mathrm{kcal} / \mathrm{mol}$ (equal to $-2.327 \mathrm{kcal} / \mathrm{mol}+7.055 \mathrm{kcal} / \mathrm{mol}$ ) for this complex. 3) Based on above assumption and computation, we set the entropy change in the process of aspirin binding to various putative targets as approximate value $4.728 \mathrm{kcal} / \mathrm{mol}$.

\section{Pathway enrichment analysis and interaction network construction}

To analyze the significance of KEGG pathways associated with our predicted targets, we collected UniProtKB accession number (AC) of these targets and performed KEGG pathway annotation using the DAVID tool (http://david.abcc.ncifcrf.gov/). The significantly overrepresented KEGG pathways were identified based on the Bonferroni-adjusted $\mathrm{P}$ value $(\mathrm{P}<0.01)$ (Huang et al. 2009). In addition, based on these pathways, an integrated targets-cellular effect interaction network was constructed.

\section{Results}

\section{Identification of putative targets of aspirin in human proteome}

We presented a proteome-wide prediction of aspirin targets using structural bioinformatics and system biology approaches. We used comparison of BSiteAs to recognize putative targets and further refined by docking and MM-PBSA in structural bioinformatics part whereas pathway enrichment analysis and interaction network construction were performed in system biology 
207

208

209

210

211

212

213

214

215

216

217

218

219

220

221

222

223

224

225

226

227

228

229

230

231

232

233

234

235

236

237

section. The steps in our pipeline for proteome-wide prediction of aspirin-binding proteins are shown in Figure 1. Firstly, the binding sites of aspirin (BSiteAs) were used as queries to search against 17425 non-redundant structures of human proteins in our self-build structure database using the program CMASA. Totally, 79 proteins with putative BSiteAs were identified (Table S1). Of these proteins, the top 10 ranked proteins are members of the phospholipase A2. cyclooxygenase, lactoperoxidase and Chitotriosidase families, which are the primary targets of aspirin. The remaining 69 proteins have different structural folds from the primary targets.

The hit proteins have similar local structures with BSiteAs and potential to bind to aspirin. However, it does not mean that aspirin can certainly bind to these proteins. In the second step, molecular docking was used to assess whether aspirin can bind to these proteins. CDOCKER in the Discovery Studio v3.1 was used to dock aspirin to the predicted binding site on these proteins. Proteins that failed to dock aspirin were removed from the target list. Only 26 proteins were considered for further analysis after filtering by molecular docking, 10 proteins of which are the primary targets of aspirin (Table S1).

Finally, MM-PBSA free energy calculation was performed for the lowest-energy proteinaspirin complex obtained in the docking step. In total, 23 proteins bind to aspirin with binding free energies $\left(\Delta \mathrm{G}_{\text {bind }}\right)<0$ listed in Table 1 and selected as the putative targets of aspirin. Next, we analyzed the binding modes and affinities of aspirin to these targets, the structural similarity and pathway enrichment of these targets and clinical outcomes of aspirin.

\section{Structural diversity of the putative targets}

In order to analyze the structural similarity of the 23 putative targets, each pair of these targets were structurally aligned using the program Combinatorial Extension (CE) (Shindyalov \& Bourne 2001). The targets were considered to be similar if the root-mean-square deviation (RMSD) between two structures is less than $4 \AA$. A network of targets was generated by linking structurally similar targets (Figure 2A). The primary targets of aspirin are colored with green, and the newly identified targets are colored with red. Only two proteins HLA class I histocompatibility antigen, A-2 alpha chain (HLA-A) and axin interactor, dorsalizationassociated protein (AIDA) clustered with the phospholipase A2 family. Proteins cyclooxygenase-1 and 2 are linked together but not similar with the other proteins. Therefore, the overall structures of the newly identified targets and the primary targets were not similar. It indicates the structural diversity of the 23 putative targets. However, the 23 targets have similar 
238 local structures. For example, proteins group IB phospholipase A2 (PLA2G1B) and cyclin239 dependent kinase 13 (CDK13) which belong to different protein families have very similar 240 binding sites of aspirin (BSiteAs) (Figure 2B).

\section{Diverse binding modes of aspirin to the putative targets}

242 In our study, aspirin was docked to the predicted binding sites on putative targets. Results of these docking experiments reveal diverse binding modes of aspirin to these targets (Figure 3). Some examples are given follows. As a first example, aspirin binds to protein CDK13 with a novel mode compared to ATP-analog inhibitor of the kinase (Figure 3A). ATP-analog inhibitors exhibit inhibitory activity of kinase by competitive binding to its ATP binding site. In contrast, aspirin binds to CDK13 in the vicinity of the ATP binding site and interact with loops (L1 and L2) which are important for ATP binding. Another example is that aspirin binds to protein rasrelated $\mathrm{C} 3$ botulinum toxin substrate 1 (RAC1), which is very different from GTP binding (Figure 3B). Aspirin binds to the other side of RACl and interact with the N-terminal part of switch II (sequence ${ }^{56} \mathrm{WDTAG}$ ), which is crucial for interaction between RAC1 and protein Arfaptin (Tarricone et al. 2001). The Arfaptin mediates cross-talk between Rac and Arf signaling pathways. The last example is that aspirin binds to protein integrin alpha-L (ITGAL) in the binding site of its inhibitor BQM (Guckian et al. 2008) (Figure 3C). The analysis of the binding mode of aspirin to the other 19 putative targets is shown in the Supplemental Figure S1-23. The statistic of 23 putative binding pockets of aspirin is shown in the Figure S24. The coordinates of 23 putative binding pockets are shown in Table S2. The size of the 23 binding pockets is ranging from 10 to 26 residues, if we define the residues lying within $5 \AA$ from aspirin as binding pockets. The pocket size of aspirin binding to phospholipase A2 (PDBID: 1OXR) and cyclooxygenase-1 (PDBID: 1PTH) is fall in this range. The most used amino acids in pockets are leucine (L), cysteine $(\mathrm{C})$, valine $(\mathrm{V})$, glycine $(\mathrm{G})$, tyrosine $(\mathrm{Y})$, isoleucine $(\mathrm{I})$, alanine $(\mathrm{A})$ and phenylalanine $(\mathrm{F})$. The eight amino acids appeared in the pockets more than 20 times. All the eight amino acids are with hydrophobic side chain except cysteine(C). It suggests that hydrophobic interaction is important for aspirin binding. Additionally, the H-bond is also important for aspirin binding because 78\% (18/23) binding pockets formed H-bonds with aspirin. The most used amino acids involved in H-bond formation are aspartic acid (D), lysine $(\mathrm{K})$ and histidine $(\mathrm{H})$. Therefore, the combinations of these charged amino acids $(\mathrm{D}, \mathrm{K}$ and $\mathrm{H})$ and hydrophobic amino acids $(\mathrm{L}, \mathrm{V}, \mathrm{G}$, Y, I, A and F) may form the pocket that aspirin prefers to bind to. 
269 The binding affinities of aspirin to putative targets

270 To obtain accurate estimate of the binding energy of different putative targets with aspirin, MM-

271 PBSA free energy calculation protocol was used. Combination of the experimental study (Singh

272 et al. 2005) and MM-PBSA free energy calculation, we obtained the estimated entropy change

$273(\mathrm{~T} \Delta \mathrm{S}=4.728 \mathrm{kcal} / \mathrm{mol})$ upon aspirin binding. The entropy changes do not have large fluctuations

274 when the same ligand binds to a different acceptor based on the study of Chang \& Gilson (Chang

$275 \&$ Gilson 2004). Therefore, the entropy changes when aspirin binds to various putative targets

276 was assumed as $4.728 \mathrm{kcal} / \mathrm{mol}$ to compare free energies associated with different aspirin

277 binding putative targets. The binding free energies including entropy change for the 23 proteins

278 binding to aspirin were calculated and listed in Table 1. The binding free energies of the 23

279 proteins with aspirin are varied from -6.0 (group IID secretory phospholipase A2, PLA2G2D) to

$280-33.0$ (exosome component 3, EXOSC3) kcal/mol. Overall, The binding free energies for newly

281 identified targets (the average $-18.4 \mathrm{kcal} / \mathrm{mol}$ ) are comparable to that for the primary targets (the

282 average $-15.3 \mathrm{kcal} / \mathrm{mol})$.

283 Pathway enrichment and interaction network of putative targets

284 Using DAVID tool, we find that our predicted targets significantly overrepresented for several 285 pathways $(\mathrm{p}<0.01)$ (Table 2$)$. Some of these pathways are strongly involved in inflammation, 286 cardiovascular disease and cancer, such as VEGF signaling, Fc epsilon RI signaling, arachidonic 287 acid metabolism, gonadotropin-releasing hormone (GnRH) signaling and MAPK signaling. To 288 illustrate the relationship between the putative targets and their cellular effect, an integrated 289 interaction network of targets-cellular effect based on their associated pathways was constructed 290 (Figure 4). The interactions between predicted targets and the major effects involved in cancer development, inflammation and cardiovascular disease were present in this network. Represented by green circles in the network, the predicted targets regulate VEGF, epsilon RI signaling, arachidonic acid metabolism, and MAPK pathways through interactions with other proteins (gray circles) connecting the pathways. Inhibition of predicted targets is expected to downregulate these pathways, and then prevent inflammation and decrease the risk of cardiovascular disease and cancer.

We use the protein RAC1 as an example to show how inhibition by aspirin leads to anti- 
298 inflammation and anti-cancer effect. RAC1, which belongs to the Rho GTPase family, can 299 regulate the machinery that controls the assembly and disassembly of cytoskeletal elements(Bid 300 et al. 2013). RAC1 is required for the VEGF-induced increase in vascular permeability 301 (Monaghan-Benson \& Burridge 2009). Increased vascular permeability is often observed in 302 inflammation(Wilhelm 1973). Therefore, RAC1 can promote the development of inflammation 303 through VEGF and Fc epsilon RI signaling pathway. Furthermore, RAC1 is involved in 304 gonadotropins expression, cytokine transcription and actin reorganization, which play a critical 305 role for proliferation, migration and evading apoptosis of cancer cell. The inhibition of RAC1 by 306 307 aspirin will attenuate these processes, which may provide an alternative explanation for the antiinflammation and cancer effect of aspirin.

308

309

310

311

312

313

314

315

316

317

318

319

320

321

322

323

324

325

326

327

328

\section{Discussion}

The overall structures of the 23 putative targets of aspirin identified in this study are very different (Figure 2A). However, the 23 targets have similar local structures. It indicates the power of the CMASA for detecting and comparing binding sites similarity from whole structures of proteins. Further, the CMASA is not only highly accurate but also sensitive and fast for detecting the binding pocket similarity ( $\mathrm{Li} \&$ Huang 2010). It can be applied in annotating and detecting similar binding pocket from two proteins which are not homologous (Flores et al. 2014; Li \& Huang 2010).

Of these targets, there are 14 targets are new and need experimental validation. We have performed literature research about aspirin and the 14 new targets. Although no direct evidence for binding of aspirin to these 14 new targets was found in the literature, possible associations with aspirin or aspirin metabolites are supported by direct or indirect evidence for half of the targets (Table 3). The seven new targets (MAPK12, ITGAL, MMP12, TNFAIP6, HLA-A, RAC1, and TNFSF14) were reported to be associated with aspirin. Slattery et.al evaluated genetic variation of MAPK12 and colon and rectal cancer risk using data from population-based case-control studies (Slattery et al. 2012). They found that for rectal cancer, aspirin users had a greater reduced risk if patients had a variant allele of MAP3K1 rs2548663 or MAPK12 rs2272857. In addition, another study reported that the gene expression of MAPK12 showed a significant down-regulation in the colorectal cancer cell line SW480 treated with $1 \mathrm{mM}$ aspirin (Dibra et al. 2010). The gene expression of MMP12, TNFAIP6 and RAC1 also showed differences between pre- and post-aspirin treatment(Choi et al. 2005; Hardwick et al. 2004; Jiang 
329 et al. 2015). In future, we will select several identified targets in this study to perform 330 experimental validation.

331 We are not sure whether the acetylation will certainly occur when aspirin exerts the effects 332 other than anti-inflammatory. The primary action mechanism of aspirin is covalent binding to 333 COX-1. There are also some evidences show aspirin may exhibit non-covalent binding (Mir et al. 334 2009; Singh et al. 2005). We analyzed the possible acetylation sites in the binding pockets of all 23 targets. We found 78\% (18/23) binding pockets have possible acetylation site if we assume that the three amino acids lysine $(\mathrm{K})$, serine $(\mathrm{S})$ and threonine $(\mathrm{T})$ can be acetylated. This analysis suggests the identified targets in our study may be acetylated after binding of aspirin.

Identification of putative targets of aspirin is restricted by the available data of the 3-D structures of human protein. We used all available structural data to construct our query database. Our query database contains totally 17425 non-redundant structures of human protein from three sources: RCSB PDB database (Rose et al. 2011), Structure atlas of human genome (SAHG) (Motono et al. 2011) and GPCR Research Database (GPCRRD) (Zhang \& Zhang 2010). With the increase number of crystal structures in the future, other new targets of aspirin may be identified. Another limitation of this study is that aspirin can bind to different targets with same or different binding sites. For example, the binding sites of aspirin to PLA2 and COX-1 are different. Therefore, it is possible to miss other potential targets to which aspirin can bind. So we used all available aspirin-protein complexes in our study.

The combination of multiple methods in structural bioinformatics and system biology can improve the prediction of protein target (Grinter \& Zou 2014). Kumar et al. used pseudoreceptorbased pharmacophore to predict probable protein targets and explore the protein structural profile of Zea mays (Kumar et al. 2014a). They suggested that the combination of total probabilities and docking energies can increase the confidence in prediction of probable protein targets using docking methods. Kumar et al. also used receptor-centric and ligand-centric methods for compound prioritization (Kumar et al. 2014b). Furthermore, they combined both inverse docking and ligand-based similarity search to predict the protein targets of kinetin(Kumar et al. 2015). In this study, we combined structural bioinformatics and inverse docking to predict the targets of aspirin. We also compared our methods with the PharmMapper tool(Liu et al. 2010). The top 20 targets identified by PharmMapper tool are shown in Table S3. The targets COX-1 and COX-1 were not found in the top 20 list. Only the member in phospholipase A2 family was found in the 
360

361

362

363

364

365

366

367

368

369

370

371

372

373

374

375

376

377

378

379

380

381

382

383

384

385

386

387

388

389

390

rank 17. Therefore, for the prediction of aspirin targets, the PharmMapper tool may be not suitable. In future, we will combine multiple available methods including Kumar et al. presented methods to further analyze the identified targets in this study.

Aspirin, as a common household drug, has been around for more than a century. Its basic mechanism of anti-inflammation is well documented (Vane 1971), yet newer beneficial effects keep on adding to ever-expanding therapeutic repertoire. Aspirin is used for the prevention of cardiovascular disease and various types of cancer (Alfonso et al. 2014; Dovizio et al. 2013; Dovizio et al. 2012; Jacobs et al. 2007). However, the molecular mechanisms underlying these effects of aspirin remain unclear. Based on the primary targets of aspirin, the traditional explanation for these effects is that both the cardioprotective and cancer preventive effects of aspirin may be attributed to inhibition of platelet activation (Alfonso et al. 2014; Dovizio et al. 2013; Su et al. 2014; Thun et al. 2012). Additionally, aspirin may restore the balance of pro- and anti-angiogenic factors released from platelet and modify tumor microenvironment via antiplatelet effect (Su et al. 2014).

Our study provides an alternative explanation for the pleiotropic effects of aspirin in various diseases. The results suggest aspirin has the capacity to modulate various targets in different protein family, such as peptidase, protein kinase, prostaglandin synthase, phospholipase. As shown in the Figure 4, aspirin can not only inhibit COX-1 and 2 but also modulate the proteins ITGAL, RAC1, MAPK12 and so on. These targets are involved in a number of biology processes, such as angiogenesis, inflammation, cell proliferation, evading apoptosis, migration (Figure 4). Therefore, the preventive effect of aspirin for cardiovascular disease and various types of cancer may be due to modulation of these proteins. Of course, additional experimental studies are needed to validate our results. Our study provides some new directions for experimental verification about this potential mechanism of action of aspirin. With more studies on the mechanisms of action in the future, the unknown side of aspirin will be uncovered.

In conclusion, we characterized the target profile of aspirin in human proteome by integrating methods from local structure detecting, molecular docking, and free energy calculation. These targets have diverse folds and are from different protein family. However, they have similar aspirin-binding pockets. The binding free energy with aspirin for newly identified targets is comparable to that for the primary targets. Pathway analysis revealed that the targets were enriched in several pathways such as VEGF signaling, Fc epsilon RI signaling and 
391

392

393

394

395

396

397

398

399

400

401

402

403

404

405

406

407

408

409

410

411

412

413

414

415

416

417

418

419

420

421

422

423

424

425

426

Arachidonic acid metabolism, which are strongly involved in inflammation, cardiovascular disease and cancer. Therefore, the predicted target profile of aspirin suggests a new explanation for the ability of aspirin to prevent these diseases. Our findings enable us to understand the magical aspirin and its efficacy of disease prevention in a systematic and global view.

\section{Acknowledgments}

We thank our colleagues, Drs. Zi-Zhang Sheng and Dong-Qiang Cheng and Yu-Qi Zhao for helpful comments on the manuscript. We also thank four anonymous reviewers for valuable comments.

\section{References}

Alfonso L, Ai G, Spitale RC, and Bhat GJ. 2014. Molecular targets of aspirin and cancer prevention. British Journal of Cancer 111:61-67. Doi 10.1038/Bjc.2014.271

Avivi D, Moshkowitz M, Detering E, and Arber N. 2012. The role of low-dose aspirin in the prevention of colorectal cancer. Expert Opinion on Therapeutic Targets 16:S51-S62. 10.1517/14728222.2011.647810

Balsinde J, Balboa MA, Insel PA, and Dennis EA. 1999. Regulation and inhibition of phospholipase A(2). Annual Review of Pharmacology and Toxicology 39:175-189.

Berger JS, Brown DL, and Becker RC. 2008. Low-dose aspirin in patients with stable cardiovascular disease: a meta-analysis. The American journal of medicine 121:43-49. 10.1016/j.amjmed.2007.10.002

Bid HK, Roberts RD, Manchanda PK, and Houghton PJ. 2013. RAC1: an emerging therapeutic option for targeting cancer angiogenesis and metastasis. Molecular Cancer Therapeutics 12:1925-1934. 10.1158/1535-7163.MCT-13-0164

Burn J, Gerdes AM, Macrae F, Mecklin JP, Moeslein G, Olschwang S, Eccles D, Evans DG, Maher ER, Bertario L, Bisgaard ML, Dunlop MG, Ho JWC, Hodgson SV, Lindblom A, Lubinski J, Morrison PJ, Murday V, Ramesar R, Side L, Scott RJ, Thomas HJW, Vasen HF, Barker G, Crawford G, Elliott F, Movahedi M, Pylvanainen K, Wijnen JT, Fodde R, Lynch HT, Mathers JC, Bishop DT, and Investigators C. 2011. Long-term effect of aspirin on cancer risk in carriers of hereditary colorectal cancer: an analysis from the CAPP2 randomised controlled trial. Lancet 378:2081-2087. Doi 10.1016/S01406736(11)61049-0

Chang CE, and Gilson MK. 2004. Free energy, entropy, and induced fit in host-guest recognition: calculations with the second-generation mining minima algorithm. Journal of the American Chemical Society 126:13156-13164. 10.1021/ja047115d

Cheong HS, Park SM, Kim MO, Park JS, Lee JY, Byun JY, Park BL, Shin HD, and Park CS. 2011. Genome-wide methylation profile of nasal polyps: relation to aspirin hypersensitivity in asthmatics. Allergy 66:637-644. 10.1111/j.1398-9995.2010.02514.x 
427 Choi S, Park HS, Cheon MS, and Lee K. 2005. Expression profile analysis of human peripheral

428

429

430

431

432

433

434

435

436

437

438

439

440

441

442

443

444

445

446

447

448

449

450

451

452

453

454

455

456

457

458

459

460

461

462

463

464

465

466

467 blood mononuclear cells in response to aspirin. Archivum Immunologiae et Therapiae Experimentalis 53:151-158.

Cross MJ, Dixelius J, Matsumoto T, and Claesson-Welsh L. 2003. VEGF-receptor signal transduction. Trends in Biochemical Sciences 28:488-494. 10.1016/S09680004(03)00193-2

Deng HY, and Fang Y. 2012. Aspirin metabolites are GPR35 agonists. Naunyn-Schmiedebergs Archives of Pharmacology 385:729-737. DOI 10.1007/s00210-012-0752-0

Dent P, Yacoub A, Fisher PB, Hagan MP, and Grant S. 2003. MAPK pathways in radiation responses. Oncogene 22:5885-5896. 10.1038/sj.onc.1206701

Dibra HK, Brown JE, Hooley P, and Nicholl ID. 2010. Aspirin and alterations in DNA repair proteins in the SW480 colorectal cancer cell line. Oncology Reports 24:37-46.

Din FVN, Valanciute A, Houde VP, Zibrova D, Green KA, Sakamoto K, Alessi DR, and Dunlop MG. 2012. Aspirin Inhibits mTOR Signaling, Activates AMP-Activated Protein Kinase, and Induces Autophagy in Colorectal Cancer Cells. Gastroenterology 142:1504-+. DOI 10.1053/j.gastro.2012.02.050

Dovizio M, Bruno A, Tacconelli S, and Patrignani P. 2013. Mode of action of aspirin as a chemopreventive agent. Prospects for Chemoprevention of Colorectal Neoplasia: Springer, 39-65.

Dovizio M, Tacconelli S, Sostres C, Ricciotti E, and Patrignani P. 2012. Mechanistic and pharmacological issues of aspirin as an anticancer agent. Pharmaceuticals (Basel) 5:1346-1371. 10.3390/ph5121346

Flores DI, Sotelo-Mundo RR, and Brizuela CA. 2014. A simple extension to the CMASA method for the prediction of catalytic residues in the presence of single point mutations. PLoS One 9:e108513. 10.1371/journal.pone.0108513

Ghooi RB, Thatte SM, and Joshi PS. 1995. The mechanism of action of aspirin--is there anything beyond cyclo-oxygenase? Medical hypotheses 44:77-80.

Grinter SZ, and Zou XQ. 2014. Challenges, Applications, and Recent Advances of ProteinLigand Docking in Structure-Based Drug Design. Molecules 19:10150-10176. 10.3390/molecules 190710150

Guckian KM, Lin EY, Silvian L, Friedman JE, Chin D, and Scott DM. 2008. Design and synthesis of a series of meta aniline-based LFA-1 ICAM inhibitors. Bioorganic \& medicinal chemistry letters 18:5249-5251. 10.1016/j.bmcl.2008.08.061

Hamberg M, Sanz A, Rodriguez MJ, Calvo AP, and Castresana C. 2003. Activation of the fatty acid alpha-dioxygenase pathway during bacterial infection of tobacco leaves. Formation of oxylipins protecting against cell death. Journal of Biological Chemistry 278:5179651805. 10.1074/jbc.M310514200

Hardwick JC, van Santen M, van den Brink GR, van Deventer SJ, and Peppelenbosch MP. 2004. DNA array analysis of the effects of aspirin on colon cancer cells: involvement of Rac1. Carcinogenesis 25:1293-1298. 10.1093/carcin/bgh118

Hassan C, Rex DK, Cooper GS, Zullo A, Launois R, and Benamouzig R. 2012. Primary 
prevention of colorectal cancer with low-dose aspirin in combination with endoscopy: a cost-effectiveness analysis. Gut 61:1172-1179. 10.1136/gutjnl-2011-300206

Huang DW, Sherman BT, and Lempicki RA. 2009. Systematic and integrative analysis of large gene lists using DAVID bioinformatics resources. Nature protocols 4:44-57. 10.1038/nprot.2008.211

Huang Y, Niu B, Gao Y, Fu L, and Li W. 2010. CD-HIT Suite: a web server for clustering and comparing biological sequences. Bioinformatics 26:680-682. 10.1093/bioinformatics/btq003

Ito M. 2002. The molecular organization of cerebellar long-term depression. Nature Reviews Neuroscience 3:896-902. 10.1038/nrn962

Jacobs EJ, Thun MJ, Bain EB, Rodriguez C, Henley SJ, and Calle EE. 2007. A large cohort study of long-term daily use of adult-strength aspirin and cancer incidence. Journal of the National Cancer Institute 99:608-615.

Jiang R, Wei L, Zhu M, Wu J, and Wang L. 2015. Aspirin Inhibits LPS-Induced Expression of PI3K/Akt, ERK, NF-kappaB, CX3CL1, and MMPs in Human Bronchial Epithelial Cells. Inflammation. 10.1007/s10753-015-0289-8

Kawakami T, and Galli SJ. 2002. Regulation of mast-cell and basophil function and survival by IgE. Nature Reviews Immunology 2:773-786. 10.1038/nri914

Kim HR, Appel S, Vetterkind S, Gangopadhyay SS, and Morgan KG. 2008. Smooth muscle signalling pathways in health and disease. Journal of Cellular and Molecular Medicine 12:2165-2180. 10.1111/j.1582-4934.2008.00552.x

Kollman PA, Massova I, Reyes C, Kuhn B, Huo SH, Chong L, Lee M, Lee T, Duan Y, Wang W, Donini O, Cieplak P, Srinivasan J, Case DA, and Cheatham TE. 2000. Calculating structures and free energies of complex molecules: Combining molecular mechanics and continuum models. Accounts of Chemical Research 33:889-897. Doi 10.1021/Ar000033j

Kuhn B, Gerber P, Schulz-Gasch T, and Stahl M. 2005. Validation and use of the MM-PBSA approach for drug discovery. Journal of medicinal chemistry 48:4040-4048. Doi $10.1021 / \mathrm{Jm} 049081 \mathrm{q}$

Kumar SP, Jha PC, Pandya HA, and Jasrai YT. 2014a. Implementation of pseudoreceptor-based pharmacophore queries in the prediction of probable protein targets: explorations in the protein structural profile of Zea mays. Molecular BioSystems 10:1833-1844. $10.1039 / \mathrm{c} 4 \mathrm{mb} 00058 \mathrm{~g}$

Kumar SP, Pandya HA, Desai VH, and Jasrai YT. 2014b. Compound prioritization from inverse docking experiment using receptor- centric and ligandcentric methods: a case study on Plasmodium falciparum Fab enzymes. Journal of Molecular Recognition 27:215-229. $10.1002 / \mathrm{jmr} .2353$

Kumar SP, Parmar VR, Jasrai YT, and Pandya HA. 2015. Prediction of protein targets of kinetin using in silico and in vitro methods: a case study on spinach seed germination mechanism. Journal of Chemical Biology 8:95-105. 10.1007/s12154-015-0135-3

Li GH, and Huang JF. 2010. CMASA: an accurate algorithm for detecting local protein structural similarity and its application to enzyme catalytic site annotation. $B M C$ 
509

510

511

512

513

514

515

516

517

518

519

520

521

522

523

524

525

526

527

528

529

530

531

532

533

534

535

536

537

538

539

540

541

542

543

544

545

546

547

548

549

Bioinformatics 11:439. 10.1186/1471-2105-11-439

Liu X, Ouyang S, Yu B, Liu Y, Huang K, Gong J, Zheng S, Li Z, Li H, and Jiang H. 2010. PharmMapper server: a web server for potential drug target identification using pharmacophore mapping approach. Nucleic Acids Research 38:W609-614. 10.1093/nar/gkq300

Mir R, Singh N, Vikram G, Kumar RP, Sinha M, Bhushan A, Kaur P, Srinivasan A, Sharma S, and Singh TP. 2009. The structural basis for the prevention of nonsteroidal antiinflammatory drug-induced gastrointestinal tract damage by the C-lobe of bovine colostrum lactoferrin. Biophysical Journal 97:3178-3186. 10.1016/j.bpj.2009.09.030

Monaghan-Benson E, and Burridge K. 2009. The regulation of vascular endothelial growth factor-induced microvascular permeability requires Rac and reactive oxygen species. Journal of Biological Chemistry 284:25602-25611. 10.1074/jbc.M109.009894

Motono C, Nakata J, Koike R, Shimizu K, Shirota M, Amemiya T, Tomii K, Nagano N, Sakaya N, Misoo K, Sato M, Kidera A, Hiroaki H, Shirai T, Kinoshita K, Noguchi T, and Ota M. 2011. SAHG, a comprehensive database of predicted structures of all human proteins. Nucleic Acids Research 39:D487-D493. Doi 10.1093/Nar/Gkq1057

Mullarkey MF, Thomas PS, Hansen JA, Webb DR, and Nisperos B. 1986. Association of aspirin-sensitive asthma with HLA-DQw2. The American review of respiratory disease 133:261-263. 10.1164/arrd.1986.133.2.261

Nagan N, and Zoeller RA. 2001. Plasmalogens: biosynthesis and functions. Progress in Lipid Research 40:199-229.

Nemerovski CW, Salinitri FD, Morbitzer KA, and Moser LR. 2012. Aspirin for Primary Prevention of Cardiovascular Disease Events. Pharmacotherapy 32:1020-1035. Doi 10.1002/Phar.1127

Otterdal K, Smith C, Oie E, Pedersen TM, Yndestad A, Stang E, Endresen K, Solum NO, Aukrust P, and Damas JK. 2006. Platelet-derived LIGHT induces inflammatory responses in endothelial cells and monocytes. Blood 108:928-935. 10.1182/blood-200509-010629

Racenis PV, Lai JL, Das AK, Mullick PC, Hajra AK, and Greenberg ML. 1992. The acyl dihydroxyacetone phosphate pathway enzymes for glycerolipid biosynthesis are present in the yeast Saccharomyces cerevisiae. Journal of Bacteriology 174:5702-5710.

Raju N, Sobieraj-Teague M, Hirsh J, O'Donnell M, and Eikelboom J. 2011. Effect of aspirin on mortality in the primary prevention of cardiovascular disease. The American journal of medicine 124:621-629. 10.1016/j.amjmed.2011.01.018

Rose PW, Beran B, Bi CX, Bluhm WF, Dimitropoulos D, Goodsell DS, Prlic A, Quesada M, Quinn GB, Westbrook JD, Young J, Yukich B, Zardecki C, Berman HM, and Bourne PE. 2011. The RCSB Protein Data Bank: redesigned web site and web services. Nucleic Acids Research 39:D392-D401. Doi 10.1093/Nar/Gkq1021

Rothwell PM, Wilson M, Elwin CE, Norrving B, Algra A, Warlow CP, and Meade TW. 2010. Long-term effect of aspirin on colorectal cancer incidence and mortality: 20-year followup of five randomised trials. Lancet 376:1741-1750. 10.1016/S0140-6736(10)61543-7 
550 Rothwell PM, Wilson M, Price JF, Belch JFF, Meade TW, and Mehta Z. 2012. Effect of daily

551

552

553

554

555

556

557

558

559

560

561

562

563

564

565

566

567

568

569

570

571

572

573

574

575

576

577

578

579

580

581

582

583

584

585

586

587

588

589

590 aspirin on risk of cancer metastasis: a study of incident cancers during randomised controlled trials. Lancet 379:1591-1601. Doi 10.1016/S0140-6736(12)60209-8

Ruf F, and Sealfon SC. 2004. Genomics view of gonadotrope signaling circuits. Trends in Endocrinology \& Metabolism 15:331-338. 10.1016/j.tem.2004.07.008

Schnell O, Erbach M, and Hummel M. 2012. Primary and secondary prevention of cardiovascular disease in diabetes with aspirin. Diabetes \& Vascular Disease Research 9:245-255. Doi 10.1177/1479164112441486

Sclabas GM, Uwagawa T, Schmidt C, Hess KR, Evans DB, Evans DB, Abbruzzese JL, and Chiao PJ. 2005. Nuclear factor kappa B activation is a potential target for preventing pancreatic carcinoma by aspirin. Cancer 103:2485-2490. Doi 10.1002/Cncr.21075

Shindyalov I, and Bourne P. 2001. CE: a resource to compute and review 3-D protein structure alignments. Nucleic Acids Research 29:228-229.

Shureiqi I, Jiang W, Zuo X, Wu Y, Stimmel JB, Leesnitzer LM, Morris JS, Fan HZ, Fischer SM, and Lippman SM. 2003. The 15-lipoxygenase-1 product 13-S-hydroxyoctadecadienoic acid down-regulates PPAR-delta to induce apoptosis in colorectal cancer cells. Proceedings of the National Academy of Sciences of the United States of America 100:9968-9973. 10.1073/pnas.1631086100

Singh RK, Ethayathulla AS, Jabeen T, Sharma S, Kaur P, and Singh TP. 2005. Aspirin induces its anti-inflammatory effects through its specific binding to phospholipase $A(2)$ : Crystal structure of the complex formed between phospholipase A(2) and aspirin at 1.9 angstrom resolution. Journal of Drug Targeting 13:113-119. Doi 10.1080/10611860400024078

Slattery ML, Lundgreen A, and Wolff RK. 2012. MAP kinase genes and colon and rectal cancer. Carcinogenesis 33:2398-2408. 10.1093/carcin/bgs305

Spector AA, Fang X, Snyder GD, and Weintraub NL. 2004. Epoxyeicosatrienoic acids (EETs): metabolism and biochemical function. Progress in Lipid Research 43:55-90.

$\mathrm{Su}$ BB, Chen JH, Shi H, Chen QQ, and Wan J. 2014. Aspirin may modify tumor microenvironment via antiplatelet effect. Medical hypotheses 83:148-150. DOI 10.1016/j.mehy.2014.05.007

Tarricone C, Xiao B, Justin N, Walker PA, Rittinger K, Gamblin SJ, and Smerdon SJ. 2001. The structural basis of Arfaptin-mediated cross-talk between Rac and Arf signalling pathways. Nature 411:215-219.

Thun MJ, Jacobs EJ, and Patrono C. 2012. The role of aspirin in cancer prevention. Nature reviews Clinical oncology 9:259-267. 10.1038/nrclinonc.2011.199

Touqui L, and Alaoui-El-Azher M. 2001. Mammalian secreted phospholipases A2 and their pathophysiological significance in inflammatory diseases. Current molecular medicine 1:739-754.

Vane JR. 1971. Inhibition of Prostaglandin Synthesis as a Mechanism of Action for Aspirin-Like Drugs. Nature-New Biology 231:232-\&.

Vane JR, and Botting RM. 2003. The mechanism of action of aspirin. Thrombosis research 110:255-258. 
591 Wilhelm DL. 1973. Mechanisms responsible for increased vascular permeability in acute 592 inflammation. Agents Actions 3:297-306.

593 Wu GS, Robertson DH, Brooks CL, and Vieth M. 2003. Detailed analysis of grid-based 594 molecular docking: A case study of CDOCKER - A CHARMm-based MD docking 595 algorithm. Journal of Computational Chemistry 24:1549-1562. Doi 10.1002/Jcc.10306

596 Younis N, Williams S, Ammori B, and Soran H. 2010. Role of aspirin in the primary prevention 597 of cardiovascular disease in diabetes mellitus: a meta-analysis. Expert Opinion on $598 \quad$ Pharmacotherapy 11:1459-1466. Doi 10.1517/14656561003792538

599 Zhang J, and Zhang Y. 2010. GPCRRD: G protein-coupled receptor spatial restraint database for $600 \quad 3 \mathrm{D}$ structure modeling and function annotation. Bioinformatics 26:3004-3005. DOI $601 \quad 10.1093 /$ bioinformatics/btq563

602

603 


\section{Figure 1 (on next page)}

The pipeline of the structural proteome-wide prediction of aspirin targets.

Starting with the binding sites of aspirin (BSiteAs), the pipeline integrates local structure detecting, molecular docking, free energy calculation, and pathway analysis. 


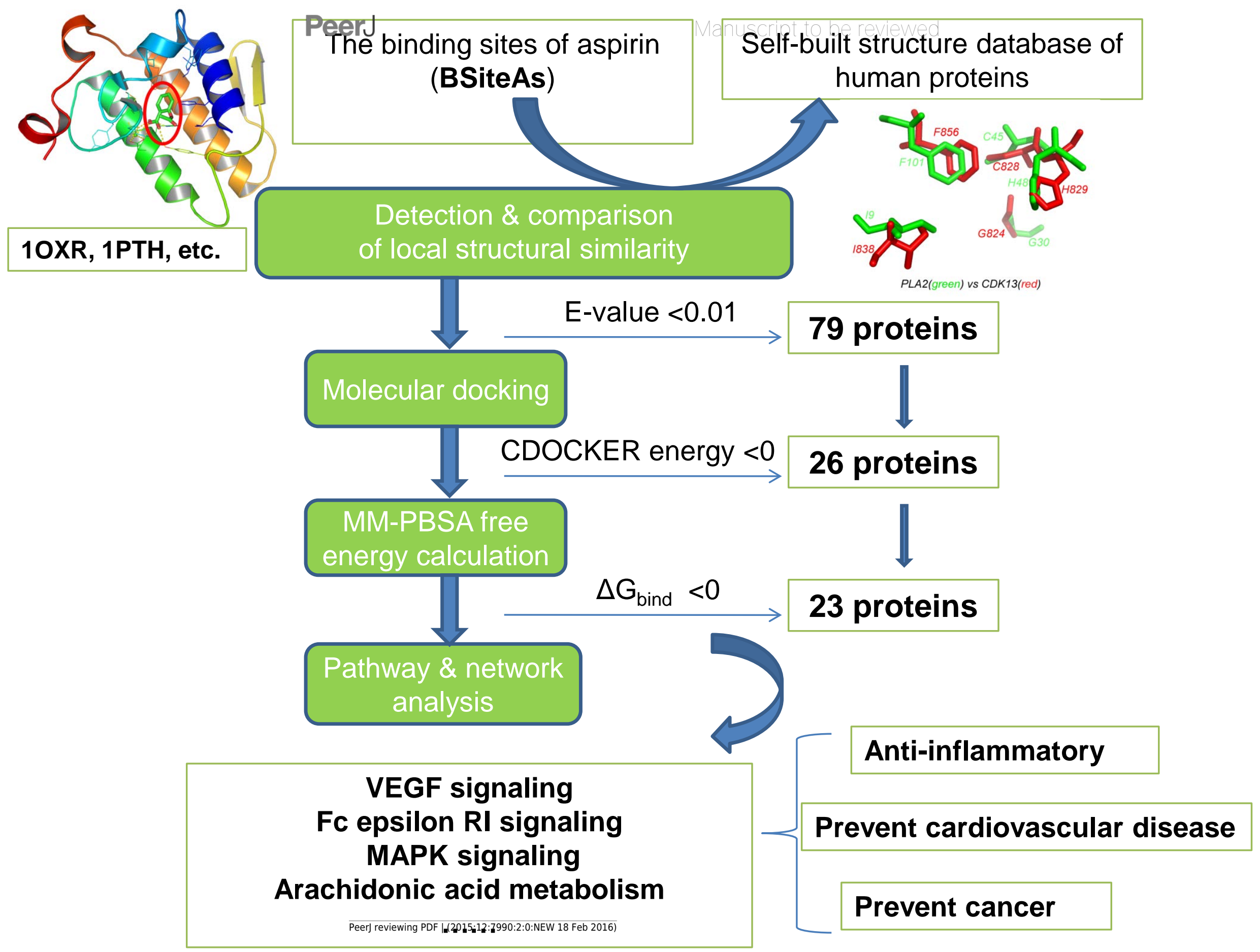


Figure 2 (on next page)

Structural diversity of the putative targets.

A) The structural similarity network of the putative targets and the structures of 10XR and 1PTH are also shown. We analyzed the structural similarity of the 23 putative targets by structural alignment. The RMSD between two linked proteins in the network is smaller than 4 $\AA$. The primary targets of aspirin are colored with green, and the newly identified targets are colored with red. B) Structural alignment of the putative binding sites of aspirin (BSiteAs) from two proteins PLA2G1B (green) and CDK13 (red). 
A
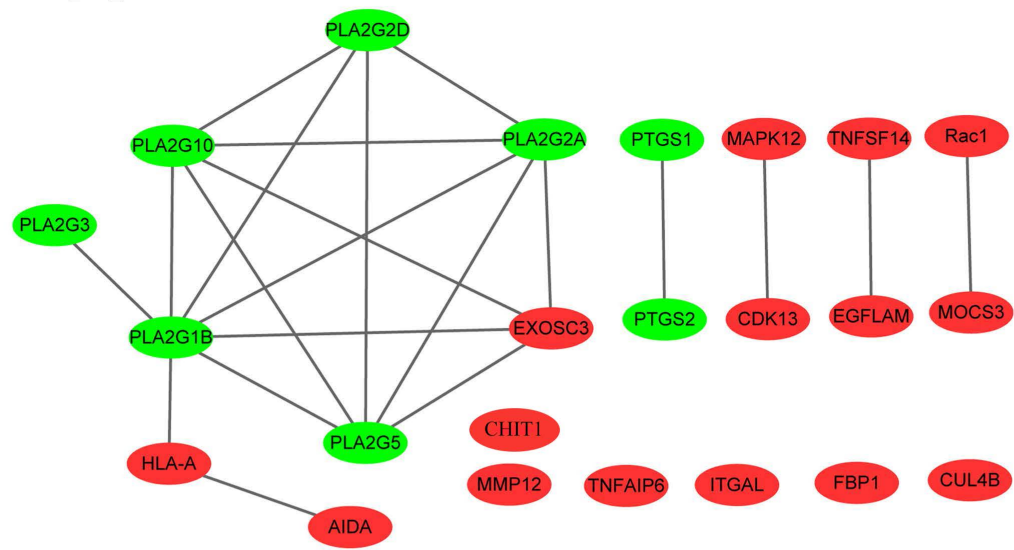

TNFAIP6 ITGAL FBP1 CUL4B
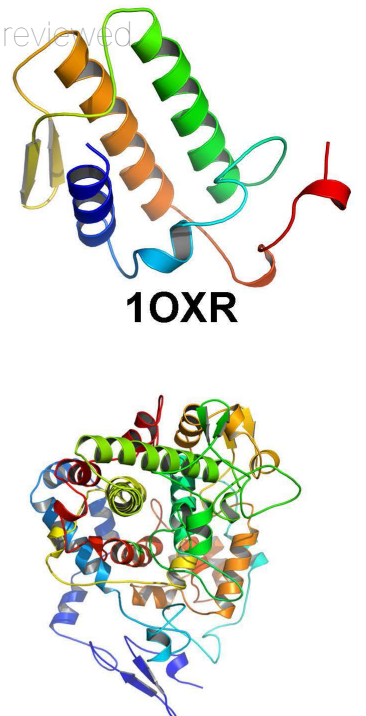

1PTH

B

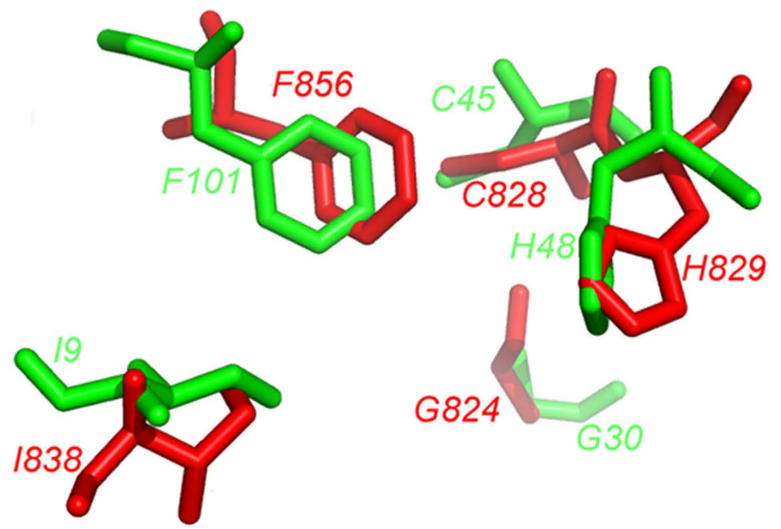

PLA2(green) VS CDK13(red) 


\section{Figure $\mathbf{3}$ (on next page)}

Diverse binding modes of aspirin to the putative targets.

The docking experiments reveal diverse binding modes of aspirin to these targets A) Aspirin binding to protein CDK13 (3LQ5.pdb). B) Aspirin binding to protein RAC1 (1RYH.pdb). C) Aspirin binding to protein ITGAL (3BQM.pdb). The overview and close-up view of the binding mode of aspirin to their putative targets are shown in top panel and bottom panel, respectively. The close-up view showed all amino acids in the vicinity of Aspirin. Aspirin and the known ligands of the three proteins are colored with green and red, respectively(top panel). The residues involved in binding to both aspirin and the ligands are shown as sticks and colored with blue (top panel). SLQ (A), GNP (B) and BQM (C) are ATP-analog inhibitor of the kinase CDK13, the substrate of the protein RAC1 and the inhibitor of protein ITGAL, respectively. 


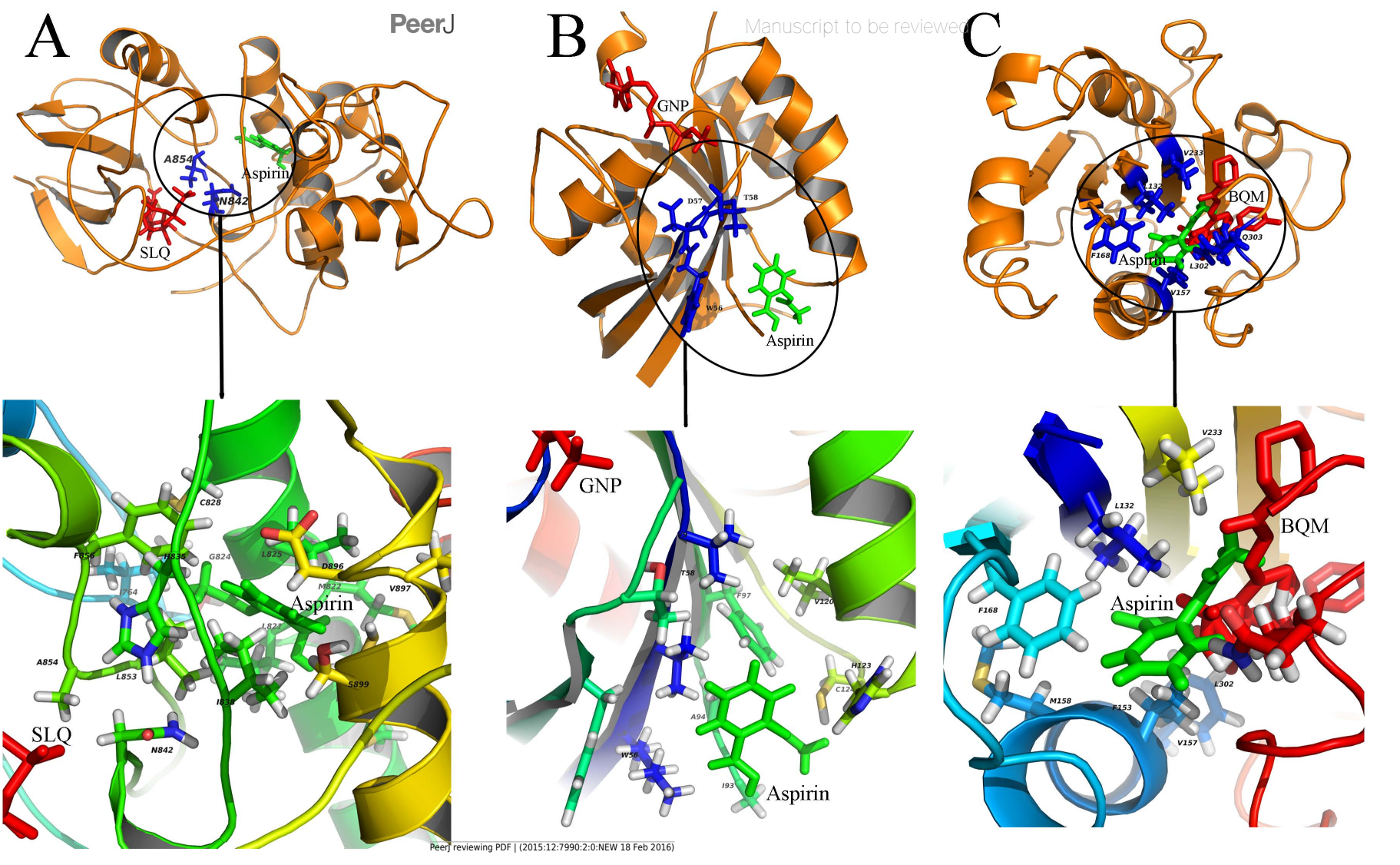


Figure 4(on next page)

An integrated interaction network of targets-cellular effect based on their associated pathways.

Predicted targets, represented by green circles in the network, regulate VEGF, epsilon RI signaling , arachidonic acid metabolism, and MAPK pathways through direct or indirect interactions with intermediate proteins (gray circles) connecting the pathways. Red squares represent cellular effects. Black lines represent activation. 


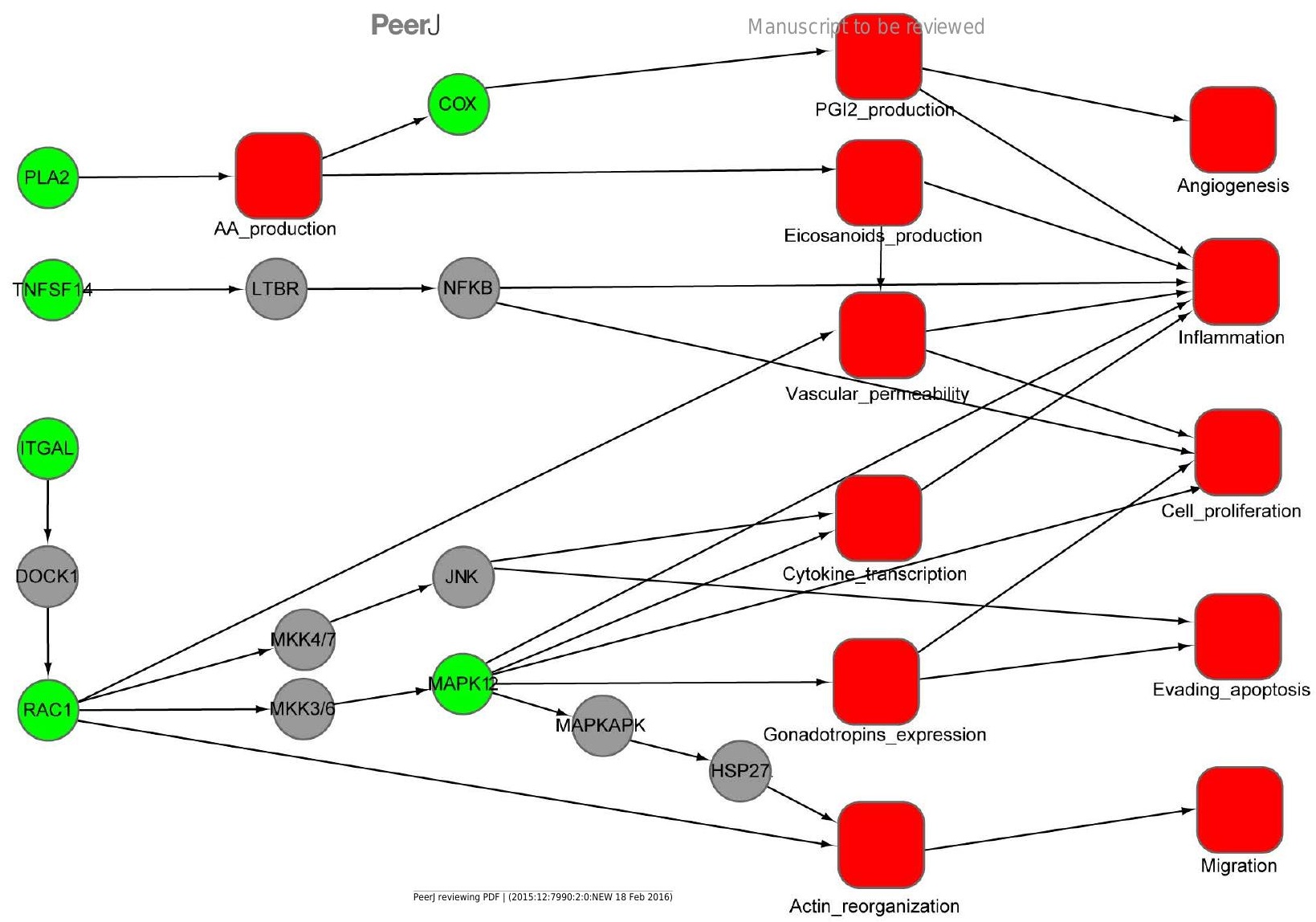




\section{Table 1 (on next page)}

The putative targets with binding free energies calculated by MM-PBSA 
1 Table 1 - The putative targets with binding free energies calculated by MM-

2 PBSA

\begin{tabular}{|c|c|c|c|}
\hline Gene name & $\begin{array}{l}\text { UniProtKB } \\
\text { entry }\end{array}$ & Family & $\begin{array}{l}\Delta G^{\mathrm{PB}} \text { binding } \\
\text { (kcal/mol) }\end{array}$ \\
\hline EXOSC3 & Q9NQT5 & RRP40 family & -33.0 \\
\hline MAPK12 & P53778 & Kinase family & -28.6 \\
\hline ITGAL & 043746 & Integrin alpha chain family & -28.0 \\
\hline PTGS2 & P35354 & Prostaglandin G/H synthase family & -20.2 \\
\hline PTGS1 & P23219 & Prostaglandin G/H synthase family & -27.6 \\
\hline PLA2G10 & O15496 & Phospholipase A2 family & -25.7 \\
\hline FBP1 & P09467 & FBPase class 1 family & -25.218 \\
\hline CUL4B & Q13620 & Cullin family & -23.0 \\
\hline MMP12 & P39900 & Peptidase M10A family & -18.6 \\
\hline CDK13 & Q14004 & kinase family & -18.4 \\
\hline TNFAIP6 & P98066 & Hyaluronan-binding protein family & -16.8 \\
\hline PLA2G3 & Q9NZ20 & Phospholipase A2 family & -14.7 \\
\hline HLA-A & O19619 & MHC class I family & -12.1 \\
\hline MOCS3 & O95396 & HesA/MoeB/ThiF family & -12.0 \\
\hline AIDA & Q96BJ3 & AIDA family & -11.1 \\
\hline RAC1 & P63000 & Rho family & -11.0 \\
\hline PLA2G5 & P39877 & Phospholipase A2 family & -10.8 \\
\hline PLA2G1B & P04054 & Phospholipase A2 family & -10.6 \\
\hline PLA2G2A & P14555 & Phospholipase A2 family & -10.6 \\
\hline TNFSF 14 & O43557 & Tumor necrosis factor family & -10.3 \\
\hline CHIT1 & Q13231 & Chitotriosidase family & -10.0 \\
\hline EGFLAM & Q63HQ2 & Pikachurin family & -9.2 \\
\hline PLA2G2D & Q9UNK4 & Phospholipase A2 family & -6.0 \\
\hline
\end{tabular}


Table 2 (on next page)

The pathways significantly overrepresented by our predicted targets $(p<0.01)$ 
1 Table 2 -The pathways significantly overrepresented by our predicted targets

$2 \quad(\mathbf{p}<\mathbf{0 . 0 1})$

\begin{tabular}{|l|l|l|l|l|}
\hline Pathway & Count & Percentage & $\begin{array}{l}\text { Adjust P- } \\
\text { value }{ }^{\text {a }}\end{array}$ & Reference \\
\hline VEGF signaling pathway & 9 & 40.9 & $4.60 \mathrm{E}-10$ & (Cross et al. 2003) \\
\hline $\begin{array}{l}\text { Arachidonic acid } \\
\text { metabolism }\end{array}$ & 8 & 36.4 & $2.00 \mathrm{E}-09$ & (Spector et al. 2004) \\
\hline $\begin{array}{l}\text { Fc epsilon RI signaling } \\
\text { pathway }\end{array}$ & 8 & 36.4 & $1.50 \mathrm{E}-08$ & $\begin{array}{l}\text { (Kawakami \& Galli } \\
\text { 2002) }\end{array}$ \\
\hline $\begin{array}{l}\text { alpha-Linolenic acid } \\
\text { metabolism }\end{array}$ & 6 & 27.3 & $1.10 \mathrm{E}-08$ & $\begin{array}{l}\text { (Hamberg et al. } \\
2003)\end{array}$ \\
\hline $\begin{array}{l}\text { Linoleic acid metabolism } \\
\text { Ether lipid metabolism }\end{array}$ & 6 & 27.3 & $1.00 \mathrm{E}-07$ & $\begin{array}{l}\text { (Shureiqi et al. } \\
\text { 2003) }\end{array}$ \\
\hline $\begin{array}{l}\text { Vascular smooth muscle } \\
\text { contraction }\end{array}$ & 6 & 27.3 & $2.80 \mathrm{E}-07$ & $\begin{array}{l}\text { (Nagan \& Zoeller } \\
\text { 2001) }\end{array}$ \\
\hline $\begin{array}{l}\text { GnRH signaling pathway } \\
\text { metabolism }\end{array}$ & 7 & 31.8 & $1.40 \mathrm{E}-06$ & $\begin{array}{l}\text { (Ruf \& Sealfon } \\
2004)\end{array}$ \\
\hline Long-term depression & 6 & 27.3 & $6.10 \mathrm{E}-06$ & $\begin{array}{l}\text { (Racenis et al. } \\
1992)\end{array}$ \\
\hline (Ito 2002)
\end{tabular}

3 a P-value was adjusted using Benjamini \& Hochberg method.

$4 \quad \mathrm{~b}$ The literature references link the refined putative targets with pathways. 


\section{Table 3 (on next page)}

Literature evidence for the association between aspirin and the new targets. 
1 Table 3. Literature evidence for the association between aspirin and the new 2 targets

\begin{tabular}{|c|c|c|c|}
\hline $\begin{array}{l}\text { Gene } \\
\text { name }\end{array}$ & Protein name & Evidence & Reference \\
\hline MAPK12 & $\begin{array}{l}\text { Mitogen-activated } \\
\text { protein kinase } 12\end{array}$ & $\begin{array}{l}\text { MAPK12 gene polymorphism } \\
\text { affect the efficacy of aspirin for } \\
\text { prevention of rectal cancer; } \\
\text { Treatment of the cell line } \\
\text { SW480 with } 1 \mathrm{mM} \text { aspirin for } \\
48 \mathrm{~h} \text { caused a significant down- } \\
\text { regulation of MAPK12 } \\
\text { expression. }\end{array}$ & $\begin{array}{l}\text { (Dibra et al. } \\
\text { 2010; Slattery } \\
\text { et al. 2012) }\end{array}$ \\
\hline ITGAL & Integrin & $\begin{array}{l}\text { ITGAL gene is hypomethylated } \\
\text { and associated with aspirin } \\
\text { hypersensitivity in asthma. }\end{array}$ & $\begin{array}{l}\text { (Cheong et al. } \\
\text { 2011) }\end{array}$ \\
\hline MMP12 & $\begin{array}{l}\text { Macrophage } \\
\text { metalloelastase }\end{array}$ & $\begin{array}{l}\text { Aspirin inhibits LPS-induced } \\
\text { MMP12 gene expression in } \\
\text { human bronchial epithelial cells. }\end{array}$ & $\begin{array}{l}\text { (Jiang et al. } \\
2015 \text { ) }\end{array}$ \\
\hline TNFAIP6 & $\begin{array}{l}\text { Tumor necrosis } \\
\text { factor-inducible } \\
\text { protein } 6\end{array}$ & $\begin{array}{l}\text { TNFAIP6 gene expression was } \\
\text { up-regulated } 2.33 \text { fold by } \\
\text { aspirin treatment in human } \\
\text { peripheral blood mononuclear } \\
\text { cells. }\end{array}$ & $\begin{array}{l}\text { (Choi et al. } \\
2005)\end{array}$ \\
\hline HLA-A & MHC class I antigen & $\begin{array}{l}\text { HLA-DQw2 allele is involved } \\
\text { in the pathogenesis of aspirin- } \\
\text { sensitive asthma. }\end{array}$ & $\begin{array}{l}\text { (Mullarkey et } \\
\text { al. 1986) }\end{array}$ \\
\hline RAC1 & Ras-related & $\mathrm{RAC} 1$ gene is highly induced in & (Hardwick et \\
\hline
\end{tabular}




\begin{tabular}{|c|c|c|c|}
\hline & $\begin{array}{l}\text { botulinum } \\
\text { substrate } 1\end{array}$ & $\begin{array}{l}\text { the HT2 colon cancer cell by } \\
\text { aspirin. This study pointed } \\
\text { towards a role for RAC1 in the } \\
\text { action of aspirin in colon } \\
\text { cancer. }\end{array}$ & al. 2004) \\
\hline TNFSF14 & $\begin{array}{l}\text { Tumor necrosis factor } \\
\text { ligand superfamily } \\
\text { member } 14\end{array}$ & $\begin{array}{l}\text { Aspirin significantly reduced } \\
\text { plasma levels of LIGHT in } 12 \\
\text { healthy controls when given for } \\
7 \text { days ( } 160 \mathrm{mg} \text { once a day). }\end{array}$ & $\begin{array}{l}\text { (Otterdal } \\
\text { al. 2006) }\end{array}$ \\
\hline
\end{tabular}

3 